University of Wollongong

Research Online

Australian Institute for Innovative Materials -

Papers

Australian Institute for Innovative Materials

$1-1-2013$

Facile synthesis of reduced graphene oxide/MWNTs nanocomposite supercapacitor materials tested as electrophoretically deposited films on glassy carbon electrodes

Widsanusan Chartarrayawadee

University of Wollongong,wc447@uow.edu.au

Simon E. Moulton

University of Wollongong, smoulton@uow.edu.au

Chee 0. Too

University of Wollongong, chee@uow.edu.au

Byung C. Kim

University of Wollongong, bkim@uow.edu.au

Rao Yepuri

University of Wollongong

See next page for additional authors

Follow this and additional works at: https://ro.uow.edu.au/aiimpapers

Part of the Engineering Commons, and the Physical Sciences and Mathematics Commons

Research Online is the open access institutional repository for the University of Wollongong. For further information contact the UOW Library: research-pubs@uow.edu.au 


\title{
Facile synthesis of reduced graphene oxide/MWNTs nanocomposite supercapacitor materials tested as electrophoretically deposited films on glassy carbon electrodes
}

\author{
Abstract \\ This paper reports on a facile synthesis method for reduced graphene oxide ( $\mathrm{rGO}$ )/multi-walled carbon \\ nanotubes (MWNTs) nanocomposites. The initial step involves the use of graphene oxide to disperse the \\ MWNTs, with subsequent reduction of the resultant graphene oxide/MWNTs composites using l-ascorbic \\ acid (LAA) as a mild reductant. Reduction by LAA preserves the interaction between the rGO sheets and \\ MWNTs. The dispersion-containing rGO/MWNTs composites was characterized and electrophoretically \\ deposited anodically onto glassy carbon electrodes to form high surface area films for capacitance \\ testing. Pseudo capacitance peaks were observed in the rGO/MWNTs composite electrodes, resulting in \\ superior performance with capacitance values up to $134.3 \mathrm{~F} \mathrm{~g}-1$ recorded. This capacitance value is \\ higher than those observed for LAA-reduced GO (LAA-rGO) $(63.5 \mathrm{~F} \mathrm{~g}-1)$, electrochemically reduced GO \\ (EC-rGO) (27.6 F g-1), or electrochemically reduced GO/MWNTs (EC-rGO/MWNTs) (98.4 F g-1)-based \\ electrodes.

\section{Keywords} \\ films, deposited, electrophoretically, tested, materials, supercapacitor, nanocomposite, mwnts, carbon, \\ oxide, graphene, reduced, synthesis, facile, electrodes, glassy \\ Disciplines \\ Engineering | Physical Sciences and Mathematics

\section{Publication Details} \\ Chartarrayawadee, W., Moulton, S. E., Too, C. O., Kim, B. C., Yepuri, R., Romeo, A. C. \& Wallace, G. G. (2013). \\ Facile synthesis of reduced graphene oxide/MWNTs nanocomposite supercapacitor materials tested as \\ electrophoretically deposited films on glassy carbon electrodes. Journal of Applied Electrochemistry, 43 \\ (9), 865-877.
}

\section{Authors}

Widsanusan Chartarrayawadee, Simon E. Moulton, Chee O. Too, Byung C. Kim, Rao Yepuri, Anthony C. Romeo, and Gordon G. Wallace 


\section{Facile synthesis of reduced graphene oxide/MWNTs nanocomposite supercapacitor materials tested as electrophoretically deposited films on glassy carbon electrodes.}

Widsanusan Chartarrayawadee ${ }^{1}$, Simon Edward Moulton ${ }^{1 *}$, Chee O. Too ${ }^{1}$, Byung Chul Kim $^{1,2}$, Rao Yepuri ${ }^{1}$, Tony Romeo ${ }^{1}$, Gordon George Wallace ${ }^{1}$

${ }^{1}$ ARC Centre of Excellence for Electromaterials Science, Intelligent Polymer Research Institute, University of Wollongong, New South Wales 2522, Australia

2 Department of Chemistry, University of Dongguk-Seoul, 30, Pil-Dong-ro, 1-gil, Jung-gu, Seoul, 100-715, Korea

\section{Abstract}

This paper reports on a facile synthesis method for reduced graphene oxide (rGO)/multiwalled carbon nanotubes (MWNTs) nanocomposites. The initial step involves the use of graphene oxide to disperse the MWNTs, with subsequent reduction of the resultant graphene oxide/MWNTs composites using L-ascorbic acid (LAA) as a mild reductant. Reduction by LAA preserves the interaction between the rGO sheets and MWNTs. The dispersion containing rGO/MWNTs composites was characterized and electrophoretically deposited anodically onto glassy carbon electrodes to form high surface area films for capacitance testing. Pseudo capacitance peaks were observed in the rGO/MWNTs composite electrodes, resulting in superior performance with capacitance values up to $134.3 \mathrm{~F} \mathrm{~g}^{-1}$ recorded. This capacitance value is higher than those observed for LAA reduced GO (LAA-rGO) (63.5 F g ${ }^{1}$ ), electrochemically reduced GO (EC-rGO) $\left(27.6 \mathrm{~F} \mathrm{~g}^{-1}\right)$ or electrochemically reduced GO/MWNTs (EC-rGO/MWNTs) (98.4 $\mathrm{F} \mathrm{g}^{-1}$ ) based electrodes.

* Corresponding author, Phone: +61-2-4221 3127. Fax: +61-2-4221 3114. e-mail: smoulton@uow.edu.au

Keywords: graphene oxide, reduced graphene oxide, carbon nanotubes, Electrophoretic deposition (EPD), nanocomposite. 


\section{Introduction}

Graphene and composites containing graphene and carbon nanotubes play an important role in the development of new electrodes for capacitors $[\mathbf{1 , 2 ]}$ and other energy storage devices [3]. The preparation of graphene from graphene oxide has been achieved via the Hummers method [4] with a reducing agent such as hydrazine [5,6], hydroquinone [7], sodium borohydride $\left(\mathrm{NaBH}_{4}\right)$ [8], or ascorbic acid (LAA) [9]. Alternatively reduction has been induced thermally [6], or through the use of sulphur-containing compounds [10] or bacteria [11].

Furthermore, it has been reported that graphene oxide serves as an effective dispersing agent for carbon nanotubes resulting in formation of a homogeneous dispersion. Tian reported that graphene oxide can be used as a dispersing agent for single-walled carbon nanotubes (SWNTs) [12] whilst Zhang used graphene oxide to assist in the aqueous dispersion of multiwalled carbon nanotubes (MWNTs) [13]. Others have prepared hybrid graphene/carbon nanotube structures using hydrazine; a highly toxic chemical and a strong reducing agent [14]. Yu produced graphene/carbon nanotube films using polymer-modified graphene sheets [15].

To form electrode structures, many approaches to deposit graphene/carbon nanotube films such as layer-by-layer [16-18] and spin coating [19,20] have been reported. Another fabrication method described in the literature is electrophoretic deposition (EPD). This is a deposition process employing the migration of colloidal particles under the influence of a DC electric field. The versatility of EPD is demonstrated in that it can be utilized to deposit solids such as metals, polymers, carbides, oxides, nitrides and glasses [21]. EPD can be classified into two types; anodic EPD and cathodic EPD [22]. When positively charged particles in 
solution move toward the negative electrode (cathode) then it is termed cathodic electrophoretic deposition. Conversely, anodic electrophoretic deposition is the movement of negatively charged particles in solution toward the positive electrode (anode). In general, the mechanism of EPD can be classified into two steps. The first step involves the movement of colloidal particles under an electric field toward the electrode. The second step involves deposition of the colloidal particles on the electrode surface to form a uniform film. EPD has proven useful in many applications such as the assembly of nanoparticles to form nanoparticle films [23-25] solid oxide fuel cell (SOFC) fabrication [26], hybrid material synthesis [27], and graphene oxide [28].

In this work we have developed a facile route to produce reduced graphene oxide (rGO)/multi-walled carbon nanotubes (MWNTs) nanocomposites. The protocol developed does not require the use of surfactants to stabilise the dispersion and does not use toxic chemicals, such as hydrazine, to induce the reduction of graphene oxide. L-ascorbic acid (LAA) has been selected as a chemical reducing agent for graphene oxide because it encourages a mild reduction [9]. The use of this reductant ensures that the interaction between the rGO and the MWNTs is maintained, thus resulting in a good dispersion needed for subsequent anodic electrophoretic deposition. These materials were then electrophoretically deposited (EPD) anodically onto glassy carbon (GC) plates to form high surface area films and tested for electrochemical capacitance. For comparison, graphene oxide and graphene oxide/MWNTs composites were EPD onto GC plates and then electrochemically reduced before testing for electrochemical capacitance. 


\section{Experimental}

\subsection{Graphene oxide (GO) synthesis}

Natural graphite (SP-1, Bay Carbon) was treated in strong acid as reported in a modified Hummers method [4]. Graphene oxide (GO) aqueous dispersion was prepared as outlined by Li et al [5,29]. The obtained graphite oxide was dispersed in ultrapure Milli-Q water resulting in a dark brown suspension. Graphite oxide was purified by washing with Milli-Q water until the $\mathrm{pH}$ became neutral and then dispersed in water to obtain a $0.05 \mathrm{wt} \%$ dispersion. Graphite oxide was exfoliated to graphene oxide by ultrasonication of the dispersion using a Branson Digital Sonifier (S450D, $500 \mathrm{~W}, 30 \%$ amplitude) for $30 \mathrm{~min}$. The light brown suspension (graphene oxide) was then separated from the un-exfoliated graphite oxide by centrifugation at 4,400 rpm for 30 min using an Eppendorf 5702 centrifuge with a rotor radius of $14 \mathrm{~cm}$.

\subsection{Purification of MWNTs}

MWNTs (Chengdu Institute of Technology, China) were heated in an oven at $200{ }^{\circ} \mathrm{C}$ for 24 hrs to oxidize the metal impurities before being dispersed in concentrated hydrochloric acid and sonicated in a sonication bath for 1.5 hrs to dissolve the impurities. After acid purification, the MWNTs were washed with Milli-Q water for several times to remove the residual $\mathrm{HCl}$ until the $\mathrm{pH}$ reached 7.

\subsection{Synthesis of graphene oxide/ MWNTs composite (GO/MWNTs)}

MWNTs (0.01 g) were dispersed into $20 \mathrm{ml}$ graphene oxide (0.05 wt \%) solution by ultrasonication using a Branson Digital Sonifier (S450D, $500 \mathrm{~W}, 30 \%$ amplitude) for $1 \mathrm{hr}$. The light brown suspension (graphene oxide) turned to a dark suspension after sonication. This dispersion was purified as per the method reported by Zhang [13] as follows. The dispersion was centrifuged for $30 \mathrm{~min}$ at $8000 \mathrm{rpm}$ to remove the unstabilized MWNTs, 
followed by centrifugation at $14000 \mathrm{rpm}$ for $20 \mathrm{~min}$ to separate the GO/MWNTs from excess GO. Finally, the dispersion was washed with Milli-Q water and centrifuged again at 14000 rpm for 20 min to obtain high purity GO/MWNTs.

\subsection{Chemical reduction of GO and GO/MWNTs composites}

L-ascorbic acid (LAA) reduced GO and L-ascorbic acid reduced GO/MWNTs, were obtained through the chemical reduction of GO and GO/MWNTs using L-ascorbic acid as reported previously by Zhang [9] L-ascorbic acid (10, 20 and $30 \mathrm{mg}$ ) was added to $10 \mathrm{~mL}$ of GO and GO/MWNTs dispersions $\left(0.1 \mathrm{mg} \mathrm{mL}^{-1}\right)$ and stirred for 48 hrs. These amounts of LAA correspond to a final LAA concentration of 5.7, 11.4 and $17.1 \mathrm{mM}$ respectively in the $10 \mathrm{~mL}$ of solvent used. The sediment composite was washed with water until the $\mathrm{pH}$ reached neutral and then redispersed in dimethyl formamide (DMF) with light sonication in a low energy sonication bath for $1 \mathrm{hr}$ to obtain a homogeneous dispersion. The dispersion in DMF was stable and can be kept for several months without aggregation and precipitation occurring. The LAA reduced GO and GO/MWNT are from here on in the paper referred to as LAA-rGO and LAA-rGO/MWNTs to indicate the conversion from the precursor graphene oxide (GO) to reduced graphene oxide(rGO).

\subsection{Anodic electrophoretic deposition (EPD) of LAA-rGO, LAA-rGO/MWNTs, GO and GO/MWNTs}

The anodic EPD of LAA-rGO and LAA-rGO/MWNTs was achieved on glassy carbon plates under ambient conditions in a two-electrode cell using a potentiostat (eDAQ) EA 160 electrochemical system (eDAQ Pty Ltd). LAA-rGO and LAA-rGO/MWNTs were EPD onto glassy carbon plates at $4.0 \mathrm{~V}$ optimised for $90 \mathrm{~s}$ to obtain a complete coverage. The EPD of GO and GO/MWNTs aqueous dispersions (not treated with LAA) on GC plates and LAA- 
rGO/MWNTs on ITO coated glass was carried out with the same electrochemical instrumentation indicated above at $4.0 \mathrm{~V}$ optimised for $30 \mathrm{~s}$ to obtain a complete coverage.

\subsection{Electrochemical reduction of GO and GO/MWNTs}

Electrochemical reduction of GO (here after referred to as EC-rGO) and GO/MWNTs (here after referred to as EC-rGO/MWNTs) as EPD films was performed using cyclic voltammetry. Initially the GO and GO/MWNTs electrodes were prepared following the same EPD procedure outlined in section 2.5. Electrochemical reduction was performed via cyclic voltammetry (CV) in a three-electrode cell with a GO or GO/MWNTs working electrode, Pt mesh counter electrode and an $\mathrm{Ag} / \mathrm{AgCl}$ reference electrode with a $3 \mathrm{M} \mathrm{NaCl}$ salt bridge. The CVs were scanned over a potential window of $0.0 \mathrm{~V}$ to $-1.3 \mathrm{~V}$ at a scan rate of $50 \mathrm{mV} \mathrm{s}^{-1}$ for 5 cycles. All CVs were performed using a potentiostat (eDAQ) EA 160 electrochemical system (eDAQ Pty Ltd). The electrolyte was 0.1 M phosphate buffered saline (PBS) of pH 7.4 [30].

\subsection{Electrochemical capacitance of LAA-rGO, LAA-rGO/MWNTs, EC-rGO and EC-rGO/MWNTs}

To study the electrochemical capacitance of LAA-rGO, LAA-rGO/MWNTs, EC-rGO and EC-rGO/MWNTs, all electrodes were subjected to cyclic voltammetry in $\mathrm{N}_{2}$ gas saturated sulphuric acid (1.0 M). Cyclic voltammetry (CV) of these electrodes was carried out in a three-electrode cell, with the LAA-rGO, LAA-rGO/MWNTs, EC-rGO and EC-rGO/MWNTs as working electrode, together with a Pt mesh counter electrode and an Ag/AgCl reference electrode with a $3 \mathrm{M} \mathrm{NaCl}$ salt bridge, at a scan rate of $10,20,30,40,50$ and $100 \mathrm{mV} \mathrm{s}^{-1}$ and over a potential range of $0.0 \mathrm{~V}$ to $+1.0 \mathrm{~V}$. All CVs were performed using a potentiostat (eDAQ) EA 160 electrochemical system (eDAQ Pty Ltd). 
Galvanostatic charge/discharge test of LAA-rGO, LAA-rGO/MWNTs, EC-rGO and ECrGO/MWNTs electrodes was carried out in a two-electrode cell with LAA-rGO, LAArGO/MWNTs, EC-rGO and EC-rGO/MWNTs electrodes used as both working and counter electrodes in each experiment at current densities of $0.1,0.2,0.4$ and $1 \mathrm{~A} \mathrm{~g}^{-1}$ with voltages between 0 and $0.6 \mathrm{~V}$ in $\mathrm{N}_{2}$ saturated sulfuric acid (1.0 M). All galvanostatic charge/discharge curves were performed using a potentiostat (eDAQ) EA 160 electrochemical system (eDAQ Pty Ltd). Electrode loadings (mass) used for electrochemical capacitance measurements were determined using a digital micro-analytical balance.

\subsection{Electrode Characterization}

The microstructure of GO/MWNTs and LAA-rGO/MWNTs dispersions, LAA-rGO, LAArGO/MWNTs, EC-rGO and EC-rGO/MWNTs film electrodes were observed by JEOL JSM7500FA cold-Field Emission Gun Scanning Electron Microscope (FEGSEM) at specific voltages of $5 \mathrm{kV}$. Optical images were obtained using a Leica DMED optical microscope controlled by Leica software version (2.4.0 R1). Spectroscopic analysis of GO, GO/MWNTs, LAA-rGO and LAA-rGO/MWNTs was performed using UV-vis spectroscopy (Model Shimadzu UV-1601 spectrophotometer with UVProbe (v.2.10) software) and Raman spectroscopy (Model Jobin Yvon Horiba HR800 Raman spectrometer with LabSpec software). X-ray photoelectron spectroscopy (XPS) analyses of GO, GO/MWNTs, LAArGO, and LAA-rGO/MWNTs were performed by the Solid State and Elemental Analysis Unit of the University of New South Wales, Australia.

\section{Results and discussions}

\subsection{GO/MWNTs Dispersion}

To prepare a composite of GO and MWNTs, aqueous GO dispersion 0.05 wt \% (20 ml) was 
ultrasonicated with MWNTs $0.01 \mathrm{~g}$ (ratio 1:1). After sonication, the dispersion had changed from the typical GO brown colour to a black colour; indicating the formation of a homogeneous dispersion of GO/MWNTs. This dispersion was stable for several months without any precipitation being observed. However, increasing the ratio of MWNTs to GO to 2:1 resulted in the agglomeration of the dispersion. This is most likely due to the increase in MWNTs overcoming the stabilizing effect of the GO. The UV-vis spectrum of a GO solution (Figure 1) shows a peak centred at a maximum wavelength of $227 \mathrm{~nm}$, indicating $\pi-\pi$ transitions of aromatic C-C bonds while a shoulder peak at $300 \mathrm{~nm}$ can be assigned to $\mathrm{n}-\pi$ transitions of $\mathrm{C}=\mathrm{O}$ bonds (Figure $1 \mathrm{a}$ ) that correspond to previously published results $[5,9,13]$. The UV-vis spectrum of GO/MWNTs (Figure 1b) shows a bathochromic shift, from 227 to $236 \mathrm{~nm}$ (as indicated by the arrow). This can be explained by interactions between the aromatic carbon of graphene oxide sheets and the aromatic side-walls of MWNTs through $\pi$ stacking [13].

\subsection{Reduction of GO and GO/MWNTs dispersions and characterization}

The reduction of GO and GO/MWNTs was carried out using L-ascorbic acid (LAA) [9]. The mild reductive ability of LAA should lead to de-epoxidation of GO sheets while maintaining the stability of the interactions of the GO sheets's aromatic regions and the side-walls of the MWNTs. The reduction of GO aqueous dispersion using different amounts of LAA for 48 hrs is shown in Figure 2a. The peak centred at the maximum wavelength of $227 \mathrm{~nm}$ for GO shifted to 259, 262 and $264 \mathrm{~nm}$ (as indicated by the arrow) when treated with 10, 20 and 30 $\mathrm{mg}(5.7,11.4$ and $17.1 \mathrm{mM})$ of LAA, respectively. The absorption intensity of the spectra is observed to increase as more LAA was used. This indicated a greater degree of reduction of GO. Therefore, this shows that LAA can be used as a mild chemical reducing agent for GO. However, our reduced GO may contain residual epoxide groups because the UV-vis spectra 
should show a redshift towards $270 \mathrm{~nm}$ for chemically reduced graphene oxide as reported by $\mathrm{Li}$ [5] LAA reduction was repeated on the GO/MWNTs aqueous dispersion to obtain rGO/MWNTs composites. It can be seen in Figure 2b that the LAA reduced GO/MWNTs (LAA-rGO/MWNTs) also shows the bathochromic shift from $236 \mathrm{~nm}$ to 265, 265 and 266 $\mathrm{nm}$ (as indicated by the arrow) at 10, 20 and $30 \mathrm{mg}(5.7,11.4$ and $17.1 \mathrm{mM})$ of LAA, respectively. However, there is a small shoulder at $236 \mathrm{~nm}$ which may be due to unreduced GO/MWNTs left in the solution due to LAA being a mild reducing agent and therefore complete reduction was not achieved.

The Raman spectra (Figure 3) of GO, LAA-rGO, GO/MWNTs and LAA-rGO/MWNTs show a higher D band peak than G band peak due to defects [31] from the acid oxidation of GO in the Hummers method. In addition, the broadened D and G bands of GO indicate a higher disordered structure [32]. MWNTs show a narrower D band, which may be due to the higher ordered structure of nanotubes compared with the fragmented GO. The 2D band of GO/MWNTs and LAA-rGO/MWNTs composites is attributed to the MWNTs, as there is no absorption for GO at the high excitation wavelength [12]. In addition, the D and G band peak shapes of GO/MWNTs and LAA-rGO/MWNTs were dominated by MWNTs, as evidenced by the narrower D band peak compared to GO and LAA-rGO, and similar G band peak shape to MWNTs [12]. However, the G band peaks of GO/MWNTs and LAA-rGO/MWNTs at all conditions seem to show splitting into two peaks due to the presence of two species; i.e., GO and MWNTs or rGO and MWNTs.

Furthermore, we know that the ratio of the $D$ to $G$ band's intensities in Raman spectra represent defects and disorder in the carbon structure [8,31]. It can be seen that the ratio of $\mathrm{D}$ to $G$ band increases after reduction, which represents an increase in the defects (Figure 3) 
which corresponds to work published by Shin [8]. This result is controversial, because we assume that double bond formation after reduction should decrease the defects. Also, the shift in D and G band peaks toward lower wavelengths is evidence to support the reduction of graphene oxide [33]. As shown in Figure 3b, both D and G bands were shifted to lower wavelengths, compared to GO. However, shifting to the lower wavelength in GO/MWNTs might be because of the interaction between aromatic carbons in GO sheets and the side-walls of MWNTs through $\pi$-stacking interactions, as discussed above.

Figure 4a to d show XPS spectra of C 1s of GO and GO/MWNTs before and after reduction with LAA for 48 hrs, respectively. There are five different peaks at 284.5, 285.1, 286.7, 288.2 and $289.1 \mathrm{eV}$ attributable to $\mathrm{C}=\mathrm{C}$ (aromatic hydrocarbon), C-C/C-H (aromatic hydrocarbon), C-O (epoxy and alkoxy), $\mathrm{C}=\mathrm{O}$ (carbonyl group) and $\mathrm{O}=\mathrm{C}-\mathrm{O}$ (carboxylic acid group) bonds. Compared to the $\mathrm{C}=\mathrm{C}$ and $\mathrm{C}-\mathrm{C} / \mathrm{C}-\mathrm{H}$ peaks, it can be seen that all $\mathrm{C}$ 1s spectra of bonding to oxygen decreased after reduction for 48 hrs. Furthermore, the C 1s spectrum of C-O showed the largest decrease after reduction. This suggests that LAA can be used as a mild reducing agent for both GO and GO/MWNTs.

Furthermore, GO/MWNTs seem to be better reduced by LAA than GO under the same conditions, as we can see the sharply decreased C 1s spectra of C-O bond (C 1s C in Figure $4 \mathrm{~b}$ and $\mathrm{d})$. It can be seen that the relative ratio of peak $\mathrm{A}(\mathrm{C}=\mathrm{C})$ to peak $\mathrm{C}(\mathrm{C}-\mathrm{O})$ of $\mathrm{GO}$ and GO/MWNTs increased from 1.7 to 3.5 and 1.3 to 5.9 respectively after being reduced by LAA for 48 hrs. When comparing between LAA-rGO and LAA-rGO/MWNTs, the ratio of peak A to peak $C$ increased from 3.5 to 5.9. Relative ratios of peak A $(C=C)$ to peak $C(C-O)$ of GO and GO/MWNTs before and after reduction by LAA for 48 hrs are listed in Table 1. The increase in ratio clearly indicates the reduction of the oxygen species after LAA 
treatment, confirming reduction has occurred.

Transmission Electron Microscopy (TEM) images of unpurified GO/MWNTs (Figure 5a and b) clearly show non-associated graphite sheets and MWNTs with little to no interaction evident. After purification and reduction of GO/MWNTs, we cannot observe free rGO sheets in the TEM images (Figure 5c to f). If the interaction was broken during reduction, the TEM images should show free rGO sheets released from the LAA-rGO/MWNTs. TEM images of purified GO/MWNTs (Figure 5c and d) and LAA-rGO/MWNT (Figure 5e and f) show that LAA can maintain the interaction between rGO and MWNTs. The TEM image of LAArGO/MWNTs after reduction shows the coexistence of these two species, without separation or loss of interaction of rGO and MWNTs.

After reduction for 48 hrs, LAA-rGO and LAA-rGO/MWNTs were washed with ultrapure Milli-Q water and centrifuged several times to remove residual LAA from the reduction process, and then redispersed in DMF. The LAA-rGO and LAA-rGO/MWNTs composites redispersed in DMF produced homogeneous dispersions that exhibited good stability. The zeta potential of LAA-rGO and LAA-rGO/MWNTs in DMF was investigated to determine the stability and charge of the particles for EPD. LAA-rGO and LAArGO/MWNTs had zeta potentials of -25.8 and $-25.3 \mathrm{mV}$ respectively in DMF. These values are very similar to the zeta potentials of GO and GO/MWNTs in water that were found to be -24.9 and $-32.2 \mathrm{mV}$ respectively. This suggests that the colloidal particles of LAA-rGO and LAA-rGO/MWNTs are quite stable in DMF and also the negative charge on LAA-rGO and LAA-rGO/MWNTs makes them suitable for electrode fabrication by EPD.

\subsection{Electrode fabrication using anodic electrophoretic deposition (EPD)}


Electrophoretic deposition (EPD) involves the migration of colloidal particles under the influence of a DC electric field. To confirm the interaction between rGO and MWNTs of rGO/MWNT composite after being reduced by LAA, solutions of LAA-rGO/MWNTs in DMF were subjected to anodic electrophoretic deposition (EPD) at $4 \mathrm{~V}$ for $30 \mathrm{~s}$ onto ITO coated glass. The SEM images presented in Figure 6 show the deposited LAA-rGO/MWNTs with the light grey areas in the images being the ITO coated glass whilst the black areas are graphene sheets and the white fibres are the MWNTs. There are no individual rGO sheets or separated MWNTs deposited on the ITO coated glass; confirming that the interaction between rGO and MWNTs was still present after reduction.

To fabricate the LAA-rGO and LAA-rGO/MWNTs electrodes, LAA-rGO and LAArGO/MWNTs dispersions were electrophoretically deposited anodically on to glassy carbon (GC) substrates $\left(1 \times 1 \mathrm{~cm}^{2}\right)$ at $4 \mathrm{~V}$ for optimised $90 \mathrm{~s}$ to obtain complete coverage of LAArGO and LAA-rGO/MWNTs films. This optimised EPD time is longer than for EPD onto ITO above to ensure complete coverage of the GC surface. The morphology of these films was investigated by SEM. It can be seen that glassy carbon substrates were fully covered by LAA-rGO and LAA-rGO/MWNTs respectively (Figure 7a and b) compared with blank glassy carbon (Figure 7d). A high magnification SEM image at 100000x of LAArGO/MWNTs (Figure 7c) also shows the synergism between GO sheets and MWNTs; as we can see MWNTs laid down on top of and underneath the GO sheets.

The capacitance can be calculated by using Equation 1 [34];

$$
\mathrm{C}=\frac{\mathrm{Q}}{2 \mathrm{Vm}}
$$

Where $\mathrm{C}$ is the specific capacitance $\left(\mathrm{F} \mathrm{g}^{-1}\right), \mathrm{Q}$ is the charge calculated from the area under the 
anodic and cathodic current peak (mA s), $\mathrm{V}$ is the scanned potential window (V), and $\mathrm{m}$ is the mass of the composite (mg).

Cyclic voltammograms (CV) of LAA-rGO and LAA-rGO/MWNTs electrodes show good symmetry with rectangular shapes; with the $\mathrm{CV}$ at the highest scan rate $\left(100 \mathrm{mV} \mathrm{s}^{-1}\right)$ (Figure 8a and b) also showing peaks. This suggests that LAA-rGO and LAA-rGO/MWNTs electrodes show quick charge/discharge processes with pseudo capacitance behaviour from the oxygen groups on LAA-rGO and LAA-rGO/MWNTs, and transition of quinine/hydroquinone groups for carbon materials [35-38]. This can be clearly observed at higher scan rates (above $30 \mathrm{mV} \mathrm{s}^{-1}$ ) resulting in higher charge and higher specific capacitance obtained as scan rates were increased (specific capacitance increased from $\underline{44.1 \pm 0.9 \mathrm{~F} \mathrm{~g}^{-1} \text { at scan rate } 10 \mathrm{mV} \mathrm{s}^{-1} \text { to } 63.5 \pm 0.2 \mathrm{~F} \mathrm{~g}^{-1} \text { at scan rate } 100 \mathrm{mV} \mathrm{s}^{-1} \text { for LAA- }}$

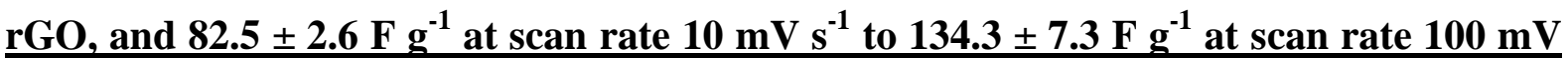
$\underline{\mathrm{s}^{-1} \text { for LAA-rGO/MWNTs; where the mass of the LAA-rGO was } 0.030 \mathrm{mg} \text { and LAA- }}$ rGO/MWNTs was 0.020 mg). As discussed previously, LAA-rGO and LAA-rGO/MWNTs were not completely reduced as shown by UV-vis spectroscopy (Figures 2a and b) and XPS spectra (Figures $4 \mathrm{~b}$ and d). So, there are oxygen functional groups left on the LAA-rGO and LAA-rGO/MWNTs that resulted in the clear pseudo capacitance peaks observed.

Since the specific capacitances of LAA-rGO and LAA-rGO/MWNTs were found to be 63.5 and $134.3 \mathrm{~F} \mathrm{~g}^{-1}$ respectively at a scan rate of $100 \mathrm{mV} \mathrm{s}^{-1}$, they confirm that added MWNTs improve the capacitance behaviour above that of the rGO electrode alone, and reduction using a mild chemical reducing agent such as LAA resulted in superior electrochemical capacitance. This specific capacitance of $134.3 \mathrm{~F} \mathrm{~g}^{-1}$ for LAA-rGO/MWNTs is higher than previous studies (in sulphuric acid electrolyte) by other workers on thermally expanded GO 
at $1050{ }^{\circ} \mathrm{C}\left(117 \mathrm{~F} \mathrm{~g}^{-1}\right)$ [39], reduced GO-SnO 2 composite (43 $\left.\mathrm{F} \mathrm{g}^{-1}\right)$ [40], polymer modified graphene/carbon nanotube hybrid film (120 $\mathrm{F} \mathrm{g}^{-1}$ ) [15], MWNTs-based supercapacitor (102 F $\left.\mathrm{g}^{-1}\right)$ [41] and graphene/MWNT/MnO 2 material $\left(126\right.$ F g $\left.^{-1}\right)$ [42].

The specific capacitance of LAA-rGO and LAA-rGO/MWNTs from the charge/discharge curves can be obtained by using Equation 2 [43].

$$
\mathrm{C}=\frac{\mathrm{I} \times \Delta \mathrm{T}}{\mathrm{m} \times \Delta \mathrm{E}}
$$

Where $\mathrm{C}\left(\mathrm{F} \mathrm{g}^{-1}\right)$ is the specific capacitance obtained from charge/discharge curves, I (A) is discharge current, $\Delta \mathrm{T}(\mathrm{s})$ is the discharge time, $\mathrm{m}(\mathrm{g})$ is mass of the composite and $\Delta \mathrm{E}(\mathrm{V})$ is the potential window. A specific capacitance of $31.7 \pm 1.1$ and $49.5 \pm 5.2 \mathrm{~F} \mathrm{~g}^{-1}$ was obtained from galvanostatic charge/discharge curves at the current density $0.1 \mathrm{Ag}^{-1}$ of LAA-rGO and LAA-rGO/MWNTs (Figure 9a and b) respectively; where the mass of the LAA-rGO was

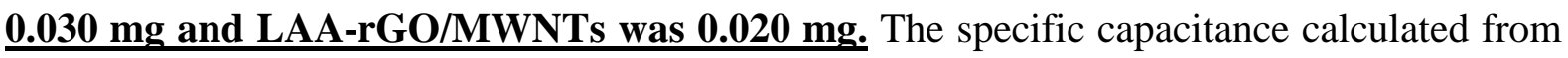
galvanostatic charge/discharge curves (two-electrode cells) is lower than the value obtained from the cyclic voltammogram (three-electrode cells) because specific capacitance was determined using different cell systems [44] and the specific capacitance obtained from Equation 2 was calculated from the discharge time only.

Further comparison of electrochemical capacitance behaviour was studied on electrochemically reduced EPD GO (here after referred to as EC-rGO) and GO/MWNTs (here after referred to as EC-rGO/MWNT) electrodes. Electrodes were fabricated by EPD of GO and GO/MWNTs aqueous dispersions on glassy carbon substrates $\left(1 \times 1 \mathrm{~cm}^{2}\right)$ at $4.0 \mathrm{~V}$ for $30 \mathrm{~s}$; as optimised for full coverage of the substrate. The complete coverage of GO and GO/MWNTs film was observed on glassy carbon as shown in Figure 10a and c respectively. 
Furthermore, there is no change in morphology or any evidence of delaminating of EC-rGO and EC-rGO/MWNTs films on glassy carbon substrates after electrochemical reduction; as shown in SEM images (Figure 10b and d).

Electrodes fabricated by EPD from GO and GO/MWNTs aqueous dispersions were subjected to reduction by cyclic voltammetry in $0.1 \mathrm{M}$ aqueous solution of PBS, $\mathrm{pH}$ 7.4. Figure 11a shows that the first cycle of the cyclic voltammogram of the electrochemical reduction of GO exhibits a broad reduction peak at approximately $-1.2 \mathrm{~V}$ while the electrochemical reduction of GO:MWNTs shows a sharp reduction peak at a potential around $-0.85 \mathrm{~V}$ (Figure 11b). The reduction current generated for the GO/MWNT film is also approximately double that for the GO film. The less cathodic electrochemical reduction potential for GO/MWNTs indicates that the electrochemical reduction process of GO/MWNTs needed less energy than GO. This can be explained by the MWNTs serving as conducting tracks in this system and promoting charge transfer in the electrochemical reduction [30]. It can also be observed that, in all cases, the reduction peak only appears during the first cycle. This indicates that the reduction of GO to rGO is completed during the first cycle and is irreversible under these conditions.

The electrochemical capacitance of EC-rGO and EC-rGO/MWNTs were further investigated. It can be seen that the CV curves for the EC-rGO electrode (Figure 12a) are symmetrical but not rectangular. This indicates that EC-rGO does not behave as an ideal capacitor and may not be as suitable for use as capacitor materials. Furthermore, the cyclic voltammograms of the EC-rGO electrodes are different from that of LAA-rGO electrodes (Figure 8a) as they show reduced pseudo capacitance peaks which might be due to the smaller amount of oxygen content left on EC-rGO (compared to the LAA-rGO) after electrochemical reduction; in keeping with reports of faradaic peak currents being observed in materials with high content 
in oxygen groups [38]. The specific capacitance at different scan rates of EC-rGO decreased as a function of scan rate; due to very little pseudo capacitance being observed at high scan rates (specific capacitance decreased from $34.3 \pm 6.2 \mathrm{~F} \mathrm{~g}^{-1}$ at scan $\underline{\text { rate } 10 \mathrm{mV} \mathrm{s}^{-1} \text { to } 27.6 \pm 3.1 \mathrm{~F} \mathrm{~g}^{-1} \text { at scan rate } 100 \mathrm{mV} \mathrm{s}^{-1} \text {, where the mass of EC-rGO was }}$

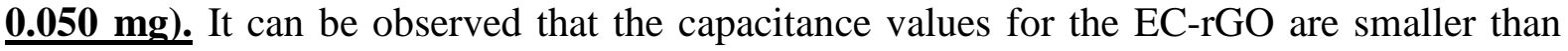
those of the LAA-rGO, suggesting that residual oxygen groups remaining on the rGO sheets are advantageous for supercapacitor applications.

In contrast, the cyclic voltammograms of EC-rGO/MWNTs (Figure 12b) show pseudo capacitance peaks, as we expected, due to the oxygen groups on the EC-rGO/MWNTs and transition of quinine/hydroquinone groups [35-38] for carbon materials as discussed previously; resulting in higher specific capacitance obtained as scan rates are increased (specific capacitance increased from $79.9 \pm 4.8 \mathrm{~F} \mathrm{~g}^{-1}$ at scan rate $10 \mathrm{mV} \mathrm{s}^{-1}$ to $98.4 \pm 6.5 \mathrm{~F}$ $\mathrm{g}^{-1}$ at scan rate $100 \mathrm{mV} \mathrm{s}{ }^{-1}$, where the mass of EC-rGO/MWNTs was $0.015 \mathrm{mg}$ ). Furthermore, a higher current density for EC-rGO/MWNTs than EC-rGO alone was also observed because of the presence of MWNTs. The presence of MWNTs in both LAArGO/MWNTs and EC-rGO/MWNTs conferred a higher current density than both LAA-rGO and EC-rGO. This can be explained in terms of the MWNTs serving as conducting wires and thus promoting charge transfer in the system [30]. In addition, MWNTs are also known to exhibit capacitive behaviour [40]. The capacitance values of the EC-rGO/MWNT are slightly lower than those of LAA-rGO/MWNT. This slight decrease may be due to the lower level of oxygen groups associated with the EC-rGO/MWNT electrode. A specific capacitance of 11.9 \pm 0.2 and $42.6 \pm 0.5 \mathrm{~F} \mathrm{~g}^{-1}$ of EC-rGO and EC-rGO/MWNTs electrodes respectively was obtained from galvanostatic charge/discharge curves (Figure 13a and b respectively) at the current density $0.1 \mathrm{Ag}^{-1}$; where the mass of EC-rGO was $0.050 \mathrm{mg}$ and EC-rGO/MWNTs 
was $0.016 \mathrm{mg}$. This can be confirmation that EC-rGO does not behave as a good capacitor and may not be suitable for use as capacitor materials. It also can be noted that the specific capacitance obtained from the LAA-rGO/MWNTs electrode is higher than that obtained from the EC-rGO/MWNTs electrode. This indicates that the LAA route is superior to the electrochemical route for obtaining rGO/MWNTs capacitor materials.

\section{Conclusions}

In conclusion, we have successfully synthesized rGO/MWNTs materials from GO/MWNTs dispersions using a facile and non-toxic method that involves mild chemical reduction using LAA that preserved the interaction between the aromatic carbon of reduced graphene oxide sheets and the side-walls of MWNTs through $\pi$-stacking interactions. LAA-rGO/MWNTs shows superior specific capacitance than EC-rGO/MWNTs, LAA-rGO and EC-rGO as shown by their respective values of 134.3, 98.4, 63.5 and $27.6 \mathrm{~F} \mathrm{~g}^{-1}$ obtained by cyclic voltammetry using a three-electrode cell. Some oxygen functional groups on GO and MWNTs remained after reduction by LAA, a mild reducing agent, that contributed to the pseudo capacitance observed in the cyclic voltammograms and are, therefore, advantageous for supercapacitor applications. The specific capacitance of $134.3 \mathrm{~F} \mathrm{~g}^{-1}$ for the LAArGO/MWNTs composite is far superior than values obtained by others for thermally expanded GO at $1050{ }^{\circ} \mathrm{C}\left(117 \mathrm{~F} \mathrm{~g}^{-1}\right)$ [39], reduced GO-SnO 2 composite (43 F $\left.\mathrm{g}^{-1}\right)$ [40], polymer modified graphene/carbon nanotube hybrid film (120 F $\left.\mathrm{g}^{-1}\right)$ [15], MWNTs-

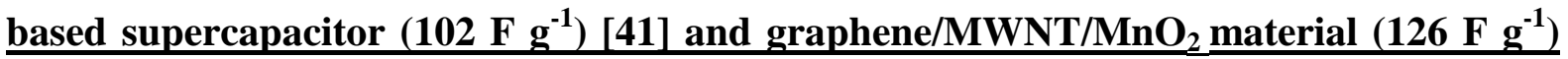
[42].

Acknowledgments 
The authors wish to thank the Australian Research Council (ARC) for their financial support. W. Chartarrayawadee wishes to thank Phayao University for his postgraduate scholarship. G. G. Wallace wishes to thank the ARC for his Laureate Fellowship and S. E. Moulton wishes to thank the ARC for his Queen Elizabeth II Fellowship. The Australian National Fabrication Facility (ANFF) is gratefully acknowledged for their support. 


\section{$\underline{\text { Captions }}$}

Table 1 Relative ratios of peak A $(\mathrm{C}=\mathrm{C})$ to peak $\mathrm{C}(\mathrm{C}-\mathrm{O})$ of $\mathrm{GO}$ and GO/MWNTs before and after reduction by LAA for 48 hrs.

Figure 1 UV visible spectra of (a) GO aqueous dispersion and (b) GO/MWNTs composite aqueous dispersion.

Figure 2 UV visible spectra of GO (a) and GO/MWNTs (b) before and after reduction by LAA for 48 hrs. The amount of LAA was varied: 10, 20 and $30 \mathrm{mg}$ (5.7, 11.4 and 17.1 $\mathrm{mM})$.

Figure 3 Raman spectra of (a) GO, MWNTs, GO/MWNTs, LAA-rGO (treated with 10, 20 and $30 \mathrm{mg}$ (5.7, 11.4 and 17.1 mM) LAA) and LAA-rGO/MWNTs (treated with 10, 20 and $30 \mathrm{mg}$ (5.7, 11.4 and $17.1 \mathrm{mM}$ ) LAA). Expansion of Raman spectra (b) of GO, GO/MWNTs and LAA-rGO, LAA-rGO/MWNTs.

Figure 4 XPS spectra of C 1s of GO (a), LAA-rGO (b), GO/MWNTs (c) and LAArGO/MWNTs (d) (treated with LAA for 48 hrs). Peaks A to E refer to C=C, C-C/C-H, C-O, $\mathrm{C}=\mathrm{O}$ and $\mathrm{O}=\mathrm{C}-\mathrm{O}$ respectively

Figure 5 TEM images at 15000x and 40000x of unpurified GO/MWNTs ( $a$ and b), purified GO/MWNTs (c and d), and purified LAA-rGO/MWNTs (e and f).

Figure 6 SEM images of electrophoretic deposited LAA-rGO/MWNTs onto ITO glass at 4.0 V for 30sec from DMF. Magnification is (a) 18000x and (b) $80000 \mathrm{x}$.

Figure 7 SEM images of EPD LAA-rGO at 13000x (a), LAA-rGO/MWNTs at 13000x (b), LAA-rGO/MWNTs at 100000x (c) and blank glassy carbon at 13000x (d).

Figure 8 Cyclic voltammograms of (a) LAA-rGO and (b) LAA-rGO/MWNTs electrodes deposited onto glassy carbon in $1.0 \mathrm{M} \mathrm{H}_{2} \mathrm{SO}_{4}$ at scan rates of 10, 20, 30, 40, 50 and $100 \mathrm{mV} \mathrm{s}^{-1}$ (Platinum mesh was used as counter electrode and $\mathrm{Ag} / \mathrm{AgCl}-3 \mathrm{M} \mathrm{NaCl}$ was used as reference electrode). Dashed arrows indicate the direction of the potential scan. 
Figure 9 Galvanostatic charge/discharge curves of (a) LAA-rGO and (b) LAArGO/MWNTs electrodes in $1.0 \mathrm{M} \mathrm{H}_{2} \mathrm{SO}_{4}$ with voltage between 0 to $0.6 \mathrm{~V}$ using a twoelectrode cell at different current densities. Current densities employed are; (1) $0.1 \mathrm{~A} \mathrm{~g}^{-1}$, (2) $0.2 \mathrm{~A} \mathrm{~g}^{-1}$, (3) $0.4 \mathrm{~A} \mathrm{~g}^{-1}$ and (4) $1.0 \mathrm{~A} \mathrm{~g}^{-1}$.

Figure 10 SEM images of GO (a), EC-rGO (b) and GO/MWNTs (c) and ECrGO/MWNTs (d). SEM magnification was 13000x.

Figure 11 Cyclic voltammograms of EPD GO (a) and GO/MWNTs (b) on a glassy carbon plate in PBS (0.1 M, pH 7.4) at a scan rate of $50 \mathrm{mV} \mathrm{s}^{-1}$ (Platinum mesh was used as counter electrode and $\mathrm{Ag} / \mathrm{AgCl}$ - $3 \mathrm{M} \mathrm{NaCl}$ was used as reference electrode). Arrows indicate the direction of the potential scan, and cyclic voltammograms of the first 3 cycles are shown.

Figure 12 Cyclic voltammograms of EC-rGO (a) and EC-rGO/MWNTs (b) electrodes in $1.0 \mathrm{M} \mathrm{H}_{2} \mathrm{SO}_{4}$ at scan rates of $10,20,30,40,50$ and $100 \mathrm{mV} \mathrm{s}^{-1}$ (Platinum mesh was used as counter electrode and $\mathrm{Ag} / \mathrm{AgCl}$ - $3 \mathrm{M} \mathrm{NaCl}$ was used as reference electrode). Arrows indicate the direction of the potential scan.

Figure 13 Galvanostatic charge/discharge curves of (a) EC-rGO and (b) ECrGO/MWNTs electrodes in $1.0 \mathrm{M} \mathrm{H}_{2} \mathrm{SO}_{4}$ with voltage between 0 to $0.6 \mathrm{~V}$ using a twoelectrode cell at different current densities. Current densities employed are; (1) $0.1 \mathrm{~A} \mathrm{~g}^{-1}$, (2) $0.2 \mathrm{~A} \mathrm{~g}^{-1}$, (3) $0.4 \mathrm{~A} \mathrm{~g}^{-1}$ and (4) $1.0 \mathrm{~A} \mathrm{~g}^{-1}$. 


\section{References}

1 Yang SY, Chang KH, Tien HW, Lee YF, Li SM, Wang YS, Wang JY, Ma CCM, Hu CC (2011) Design and tailoring of a hierarchical graphene-carbon nanotube architecture for supercapacitors. J Mater Chem 21:2374-2380

\section{$2 \quad$ J. Chen, C. Li and G. Shi, (2013) J. Phys. Chem. Lett., 4:1244-1253}

3 Cheng Q, Tang J, Ma J, Zhang H, Shinya N and Qin L-C (2011) Graphene and carbon nanotube composite electrodes for supercapacitors with ultra-high energy density. Phys. Chem. Chem. Phys., 13:17615-17624

$4 \quad$ Hummers WS, Offeman RE (1958) J Am Chem Soc 80:1339

5 Li D. Muller MB, Gilje S, Kaner RB, Wallace GG (2008) Nature Nanotech 3:101.

6 Gao X, Jang J, Nagase S (2009) J Phys Chem C 114:832.

7 Wang G, Yang J, Park J, Gou X, Wang B, Liu H, Yao J (2008) J Phys Chem C 112:8192.

8 Shin HJ, Kim KK, Benayad A, Yoon SM, Park HK, Jung IS, Jin MH, Jeong HK, Kim JM, Choi JY, Lee YH (2009) Adv Funct Mater 19:1987

9 Zhang J, Yang H, Shen G, Cheng P, Zhang J, Guo S (2010) Chem Comm 46:1112

10 Chen W, Yan L, Bangal PR (2010) J Phys Chem C 11:19885

11 E. C. Salas, Z. Sun, A. Luttge and J. M. Tour, ACS Nano, 2010, 4, 4852-4856.

12 L. Tian, M. J. Meziani, F. Lu, C. Y. Kong, L. Cao, T. J. Thorne, and Y.-P. Sun, ACS App. Mater. Interfaces, 2010, 2, 3217-3222.

13 C. Zhang, L. Ren, X. Wang and T. Liu, J. Phys. Chem. C, 2010, 114, 11435-11440.

14 V. C. Tung, L.-M. Chen, M. J. Allen, J. K. Wassei, K. Nelson, R. B. Kaner, and Y. Yang, Nano Lett., 2009, 9, 1949-1955.

15 D. Yu, and L. Dai, J. Phys. Chem. Let., 2009, 1, 467-470.

16 B. S. Shim and N. A. Kotov, Langmuir, 2005, 21, 9381-9385.

17 S.W. Lee, B.S. Kim, S. Chen, Y. Shao-Horn and P. T. Hammond, J. Am. Chem. Soc., 2009, 131, 671-679.

18 J. Shen, Y. Hu, C. Li, C. Qin, M. Shi and M. Ye, Langmuir, 2009, 525, 6122-6128.

19 S. Watcharotone, D. A. Dikin, S. Stankovich, R. Piner, I. Jung, G. H. B. Dommett, G. Evmenenko, S. E. Wu, S. F. Chen, C. P. Liu, S. T. Nguyen and R. S. Ruoff, Nano Lett. 2007, 7, 1888-1892.

20 P. Blake, P. D. Brimicombe, R. R. Nair, T. J. Booth, D. Jiang, F. Schedin, L. A. Ponomarenko, S. V. Morozov, H. F. Gleeson, E. W. Hill, A. K. Geim, and K. S. Novoselov, Nano Lett., 2008, 8, 1704-1708.

21 O. O. Van der Biest and L. J. Vandeperre, Annu. Rev. Mater. Sci., 1999, 29, 327-352. 
22 L. Besra and M. Liu, Prog. Mater. Sci., 2007, 52, 1-61.

23 R. C. Bailey, K. J. Stevenson and J. T. Hupp, Adv. Mater., 2000, 12, 1930-1934

24 M. Giersig and P. Mulvaney, J. Phys. Chem., 1993, 97, 6334-6336.

25 M. Giersig and P. Mulvaney, Langmuir, 1993, 9, 3408-3413.

26 T. Ishihara, K. Sato, Y. Mizuhara and Y. Takita, Chem. Lett., 1992, 21, 943-946.

27 N. Yamada, H. Shoji, Y. Kubo and S. Katayama, J. Mater. Sci., 2002, 37, 2071-2076.

28 S. Liu, J. Ou, J. Wang, X. Liu and S. Yang, J. Appl. Electrchem., 2011, 41, 881884.

29 D. Li, and R. B. Kaner, Science, 2008, 320, 1170-1171.

30 L. Qiu, X. Yang, X. Gou, W. Yang, Z.-F. Ma, G. G. Wallace, D. Li, Chemistry - A European Journal, 2010, 16, 10653-10658.

31 Y. Zhu, S. Murali, W. Cai, X. Li, J. W. Suk, J. R. Potts, and R. S. Ruoff, Adv. Mater., 2010, 22, 3906-3924.

32 K. N. Kudin, B. Ozbas, H. C. Schniepp, R. K. Prudhomme, I. A. Aksay, and R. Car, Nano Lett., 2008, 8, 36-41.

33 Z. Xu, and C. Gao, Macromolecules, 2010, 43, 6716-6723.

34 B. C. Kim, G. G. Wallace, Y. I. Yoon, J. M. Ko, C. O. Too, Synth. Met., 2009. 159, 1389-1392.

35 S. H. Aboutalebi, A. T. Chidembo, M. Salari, K. Konstantinov, D. Wexler, H. K. Liu, and S. X. Dou, Energy Environ. Sci., 2011, 4, 1855-1865.

36 Y. Chen, X. Zhang, D. Zhang, P. Yu, Y. Ma, Carbon, 2011, 49, 573-580.

37 D.-W. Wang, F. Li, J. Zhao, W. Ren, Z.-G. Chen, J. Tan, Z.-S. Wu, I. Gentle, G. Q. Lu, and H.-M. Cheng, ACS Nano, 2009, 3, 1745-1752,

38 Y.-R. Nian and H. Teng,. J. Electrochem. Soc., 2002, 149, A1008-A1014.

39 S. R. C. Vivekchand, C. S. Rout, K. S. Subrahmanyam, A. Govindaraj, and C. N. R. Rao, J. Chem. Sci., 2008, 120, 9-13.

40 F. Li, J. Song, H. Yang, S. Gan, Q. Zang, D. Han, A. Ivaska, and L. Niu, Nanotech., 2009, 20, 455602-455608.

41 N. Chunming and E.K. Sichel, App. Phys. Lett., 1997, 70, 1480.

42 L. Deng, Z. Hao, J. Wang, G. Zhu, L. Kang, Z-H. Liu, Z. Yang and Z. Wang, Electrochim. Acta, 2013, 89, 191-198.

43 X. Lu, H. Dou, B. Gao, C. Yuan, S. Yang, L. Hao, L. Shen and X. Zhang, Electrochim. Acta., 2011, 56, 5115-5121.

44 V. Khomenko, E. Frackowiak and F. Béguin, Electrochim. Acta., 2005, 50, 2499-2506. 